\title{
Progressive perceptual-motor impairment without generalized dementia: a type of cortical degenerative syndrome
}

\author{
K. Abe ${ }^{1}$, S. Yorifuji ${ }^{1}$, H. Tanabe ${ }^{2}$ and T. Yanagihara ${ }^{1}$ \\ Departments of ${ }^{1}$ Neurology and ${ }^{2}$ Psychiatry, Osaka University Medical School, 2-2 Yamadaoka, \\ Suita City, Osaka 565, Japan
}

Correspondence to: $K$. Abe at above address

\begin{abstract}
A patient developed slowly progressive clumsiness of both upper extremities with associated impairment of daily activities without loss of muscle strength or abnormal muscle tone. Her sensory function was normal in the primary modalities but was impaired in the discriminative tasks. A single photon emission computed tomography using ${ }^{123}$ I-iodoamphetamine showed the areas with reduced uptake in the postcentral cortices. Based on the analysis of her motor impairment, we conclude that clumsiness of hands results from higher sensory disturbance.
\end{abstract}

Keywords: Active touch - Exploratory movement - Motor clumsiness - Somatosensory cortex

\section{INTRODUCTION}

Slowly progressive cortical syndrome, including progressive aphasia, frontal lobe dementia and parietal degeneration, has been reported and has been termed as "asymmetric cortical degenerative syndrome" (Meslum, 1982; De Renzi, 1986; Benson et al., 1988; Neary et al., 1990; Casell et al., 1992). However, slowly progressive perceptual-motor impairment (motor clumsiness) without other focal higher cortical dysfunctions has not been reported. We report such a patient and discuss the nature and pathogenesis of this syndrome.

\section{CASE REPORT}

A 71 year old right-handed woman developed difficulties in dressing, using chopsticks and writing in 1989. These difficulties gradually deteriorated to the point where she could not carry out her daily activity as a housewife. She underwent a cranial computed tomography $(\mathrm{CT})$ scan but no abnormality was found. For a further examination, she was referred to our hospital in December 1991.

On examination, she showed no apparent weakness on either side of the body or extremities. She had normal control of volitional eye movements. Other cranial nerves were intact. Muscle tone was normal and deep tendon reflexes were equal bilaterally without pathological reflexes. There was no involuntary movement and she had no truncal ataxia. Sensory examination revealed intact primary modalities (touch, pain, temperature, vibration and passive joint sense) but she was defective in some discriminative tasks (two point discrimination, graphaesthesia, gravity sense). She achieved normal results in standard language test of aphasia (SLTA, a Japanese standardized test for aphasia). She showed a normal ability to name on the Boston naming test. She showed no difficulty in using object names in daily conversation. She had no aphasia, agnosia for objects and no extinction (neglect) was observed.

She was attentive and fully oriented with a digit span of six forward and five backward. On the Wechsler Adult Intelligence Scale (WAIS), verbal IQ was 94 (performance IQ was not examined because of motor clumsiness). She had no difficulty in discriminating faces, facial affect or emotional prosody. She could not draw a block design or complex figure, although she could recognize them. Memory was preserved with correct recall of three names immediately and $5 \mathrm{~min}$ afterwards. Rey's auditory verbal learning test (AVLT) showed 10 in maximum learning, 7 in delayed recall and 45 in recognition, which were normal for her age. Remote memory was also preserved. Word fluency was 12 for letter head and 10 for category, which was normal for her age.

Perseveration was not present. 


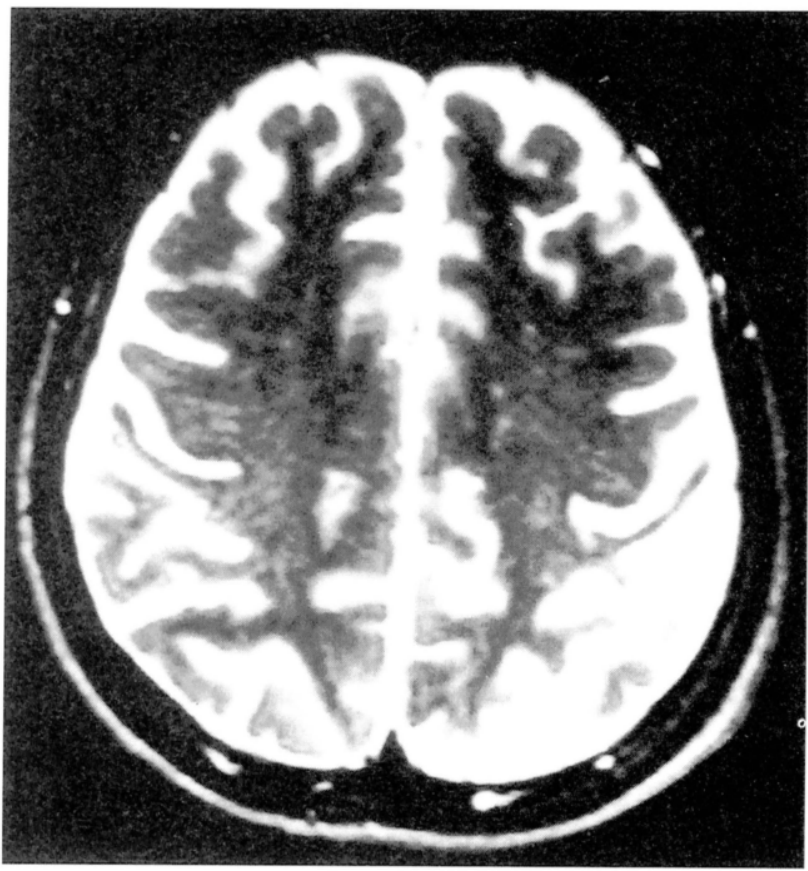

FIG. 1. Magnetic resonance images (1.5 Tesla superconducting system, spin-echo method, T2 weighted images, TR = $3000 \mathrm{~ms}$, TE $=120 \mathrm{~ms}$ ) taken January 1992 showing mild atrophy in the parietal lobes.

\section{Motor aspect}

Although she fully recognized objects, she had difficulty in manipulating objects with either of her hands, including buttoning, putting on gloves, turning a page of a book or holding a coin. These difficulties did not improve with visual help. She showed clumsiness in manipulating real objects as well as imitating the use of objects. She also showed clumsiness in gesturing, e.g. making a fist, wiggling fingers, waving goodbye, beckoning or saluting, although she fully recognized these gestures.

The ability to use a single finger on verbal command was also impaired. For instance, she could not move the index finger independently from other fingers, and demonstration by the examiner did not improve her performance. If, however, the examiner touched her finger and told her to move the touched one, the response became completely normal for each finger.

The ability to initiate finger patterns was also impaired even though she could fully recognize the finger pattern. For instance, she could not make the pattern of a "fox head" (extension of the index and the little finger and closing of the third and the fourth finger with the thumb) or a sign of money (closing of the index finger with the thumb and extending the rest).

\section{Sensory aspect}

Somatic sensation to touch, pain, temperature, vibration and passive movement was normal. There was no extinction on double simultaneous stimulation. However, two point discrimination was impaired on both hands. She could not feel the sense of motion of her own fingers when she tried to move them by herself. Weight estimation, stereognosis and perception of texture were also impaired. Graphaesthesia for numbers and identification of the direction of a line drawn on the palm were impaired.

\section{Electrophysiological examination}

An electroencephalogram was unremarkable. Somatosensory evoked potentials and nerve conduction velocities were examined bilaterally with the median, ulnar and posterior tibial nerves but showed no abnormality. A brainstem auditory evoked potential was normal.

\section{Neuroimaging}

A magnetic resonance image (MRI) showed mild atrophy in the parietal lobe and, to a lesser extent, in the frontal lobe bilaterally without abnormal intensities (Fig. 1). A magnetic resonance angiogram (MRA) showed normal cervical and intracranial vasculature. A ${ }^{123}$ I-iodoamphetamine (IMP) single photon emission computed tomography (SPECT) showed reduced isotope uptake areas in the postcentral cortex and partially in the precentral cortex (Fig. 2).

\section{Follow-up study}

She was followed-up for 4 years. A SPECT using ${ }^{123}$ I-IMP showed reduced areas of isotope uptake in the postcentral cortex and partially in the precentral cortex. The motor deficit had deteriorated so that the right hand was almost useless. However no other neuropsychological deficits had been observed.

\section{DISCUSSION}

Our patient developed motor difficulties even though she had normal motility, muscle strength and muscle tone of the individual fingers as well as intact simple dynamic coordination and gross orientation in the use of an object. She did not have involuntary movement, attentional deficit, severe cognitive deficit, memory impairment, or motivational disorder which may affect motor performances. She had normal primary sensory functions including perception of touch, pain, temperature and vibration, which were also ascertained by electrophysiological examinations. On the other hand, she had impaired percep- 


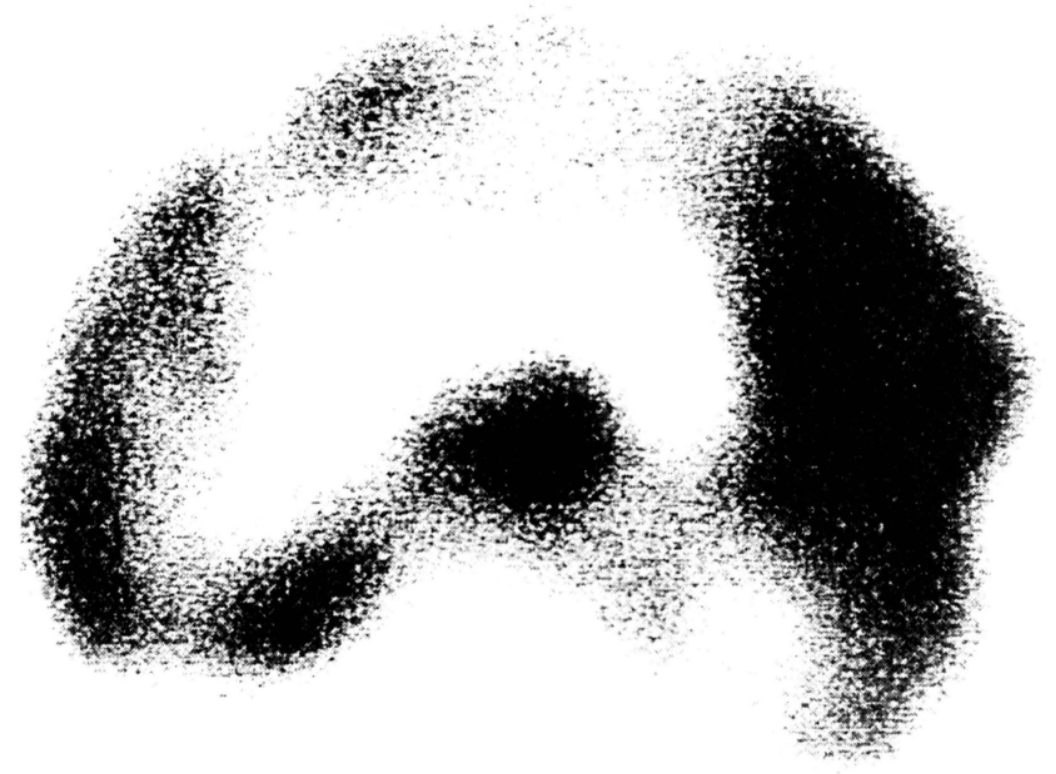

FIG. 2. Single photon emission computed tomography using 123|iodoamphetamine taken January 1992 showing reduced isotope uptake in the postcentral cortex and partially in the precentral cortex.

tion of active movement, point localization, two point discrimination, texture evaluation and stereognosis. We therefore suggest that the motor disorder in our patient may result from higher cortical sensory dysfunctions. This motor disorder may be called "limb apraxia", but the term "apraxia" may be inappropriate. We believe that "motor clumsiness secondary to cortical sensory deficits" is more suitable.

Motor clumsiness secondary to cortical sensory deficits has been demonstrated in animal experiments (Peele, 1944; Cole and Glees, 1953; Kruger and Poter, 1958; Hikosaka et al., 1985), although relatively few cases have been reported in humans with contralateral clumsiness following higher sensory disturbance (Yamadori, 1982; Montgomery, 1983; Jeannerod and Michael, 1984; Heilmann et al., 1986; Motomura et al., 1990). Motor disturbances have been observed in monkeys following postcentral lesions, including loss of ability to discriminate object shapes by active palpation (Cole and Glees, 1953), and slowness in initiation and execution of movements (Peele, 1944). Destruction of area 2 in monkeys led to the loss of finger coordination, suggesting that the postcentral cortex, especially area 2, was responsible for fine motor control (Hikosaka et al., 1985). Our patient showed clumsiness on both sides though slightly more on the right side, without showing any abnormal MRI or MRA findings, yet she had reduced isotope uptake in the postcentral cortex bilaterally on SPECT. These findings probably exclude cerebral infarction, inflammatory diseases or tumors and suggest that there was a degenerative process predominantly in the postcentral cortex.

To elucidate the pathogenesis of motor clumsiness, it is necessary to distinguish active and passive touch. Touch should be divided into touching (active touch) and being touched (passive touch) (Gibson, 1962). Active touch is not a simple combination of touch and kinesthesis but involves a perceptive system at a higher order. Norsell (1980) described active touch as starting with an intentional motor act and then proceeding with exploratory movements to make hands shape and reshape successively according to the information being collected. Disturbance of active touch may result from lesions in the parietal cortex, causing difficulty in exploratory movements because of the inability to make hands shape and reshape to grip objects (Jeannerod and Michael, 1984). Since simultaneous manifold tactile stimuli may not be meaningful without subtle sense of active movement, a loss of active touch probably has a certain role in producing astereognosis.

Although previously described patients showed improvement under visual guidance (Yamadori, 1982; Jeannerod and Michael, 1984; Motomura et al., 1990), our patient did not. According to the literature, patients who showed improvement under visual guidance had their lesions almost entirely confined to the primary sensory cortex. Since our patient had hypometabolic areas extended into the associated 
cortex, impairment of the integration between sensory and visual information might have resulted in motor clumsiness regardless of the presence or absence of visual guidance.

Several cortical degenerative diseases, including Alzheimer disease, Pick disease and corticobasal degeneration, could have caused motor clumsiness in our patient. The clinical features of our patient are different from those of Alzheimer disease. Nonetheless, Alzheimer disease can have considerable variation in the initial presentation and this possibility cannot be ruled out completely, even though she has been free of dementia or memory impairment for 4 years. Restricted cortical atrophy has been observed in some cases of Pick disease which may manifest as aphasia initially. However, atrophy in Pick disease primarily occurs in the front temporal region, while our patient has atrophy restricted to the sensory cortex. An absence of rigidity or myoclonus and symmetrical degeneration in the sensory cortex are quite atypical for corticobasal degeneration (Sawle $e t$ al., 1991). Therefore, the nature of the degenerative process in our patient remains uncertain and we must await neuropathological information. However, it is important to realize that degenerative process in the postcentral cortex can cause motor clumsiness without dementia, which should not be mistaken as other higher cortical dysfunctions.

\section{REFERENCES}

Benson DF, Davis J and Snyder BD (1988) Posterior cortical atrophy. Archives of Neurology, 45, 789-793.

Caselli RJ, Jack CR Jr, Petersen RC, Wahner HW and Yanagihara T (1992) Asymmetric cortical degenerative syndromes: Clinical and radiological correlations. Neurology, 42, 1462-1468.

Cole J and Glees P (1953) Effects of small lesions in sensory cortex in trained monkeys. Journal of Neurophysiology, 17, 1-13.
De Renzi E (1986) Slowly progressive visual agnosia or apraxia without dementia. Cortex, 22, 171-180.

Gibson JJ (1962) Observations on active touch. Psychological Review, 69, 477-491.

Heilmann KM, Rothi LG, Mack L, Feinberg $\mathrm{T}$ and Watson RT (1986) Apraxia after a superior parietal lesion. Cortex, 22, 141-150.

Hikosaka O, Tanaka M, Sakamoto $\mathbf{M}$ and Iwamura $\mathbf{Y}$ (1985) Deficits in manipulative behaviors induced by local injections of muscimol in the first somatosensory cortex of the conscious monkey. Brain Research, 325, 375380.

Jeannerod M and Michael F (1984) The control of hand movements in a case of hemianesthesia following a parietal lesion. Brain, 107, 899-920.

Kruger L and Poter P (1958) A behavioral study of functions of rolandic cortex in the monkey. Journal of Comparative Neurology, 109, 439-469.

Meslum M-M (1982) Slowly progressive aphasia without generalized dementia. Annals of Neurology, 11, 592598.

Montgomery EB (1983) Signs and symptoms from a cerebral lesion that suggest cerebellar dysfunction. Archives of Neurology, 40, 422-423.

Motomura N, Yamadori A, Asaba H, Sakai T and Sawada T (1990) Failure of manipulating objects secondary to active touch disturbance. Cortex, 26, 473-477.

Neary D, Snowden JS, Mann DMA, Northen B and Goulding PJ (1990) Frontal lobe dementia and motor neuron disease. Journal of Neurology, Neurosurgery and Psychiatry, 53, 23-32.

Norsell TL (1980) Behavioral studies of somatosensory system. Physiological Review, 60, 327-352.

Peele TL (1944) Acute and chronic parietal lobe ablations in monkeys. Journal of Neurophysiology, 7, 269-286.

Sawle GV, Brooks DJ, Marsden CD and Frackowiak RSJ (1991) Corticobasal degeneration. A unique pattern of regional cortical oxygen hypometabolism and striatal fluorodopa uptake demonstrated by positron emission tomography. Brain, 114, 541-556.

Yamadori A (1982) Palpatory apraxia. European Neurology, 21, 277-283.

(Received January 1994; accepted 16 April 1994) 


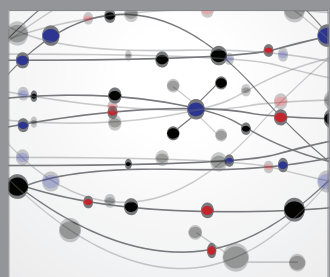

The Scientific World Journal
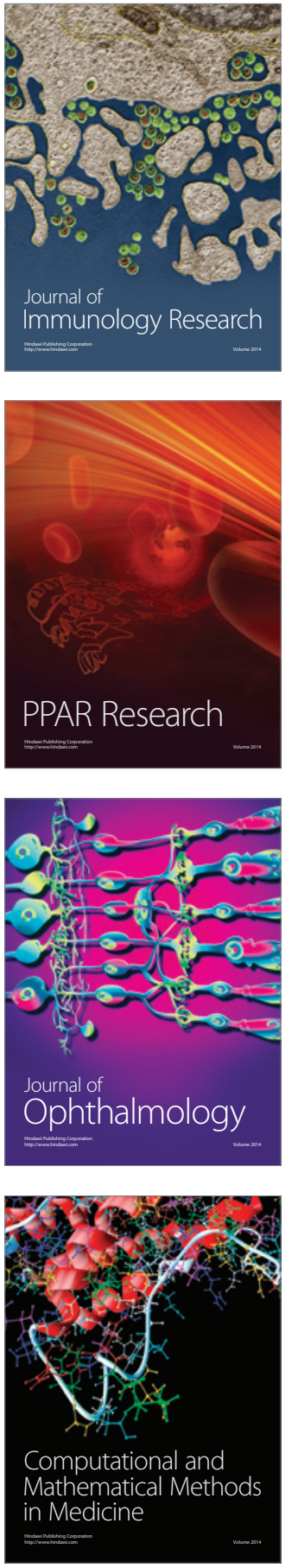

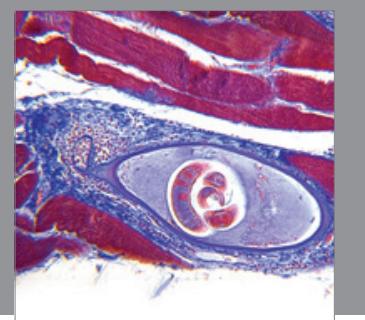

Gastroenterology

Research and Practice
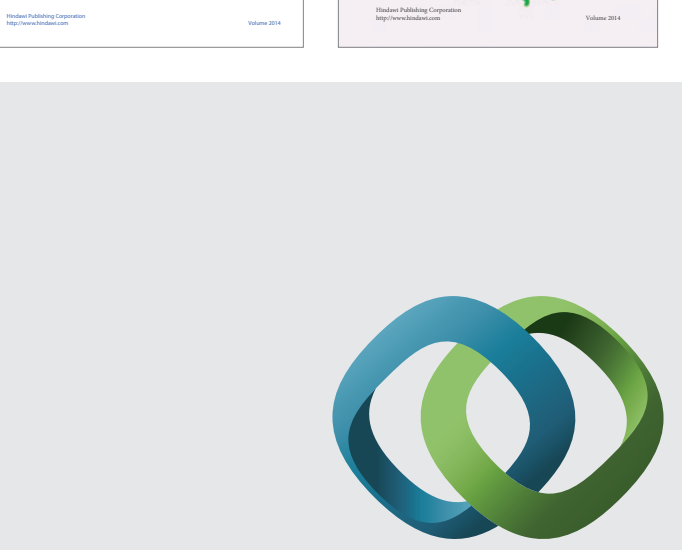

\section{Hindawi}

Submit your manuscripts at

http://www.hindawi.com
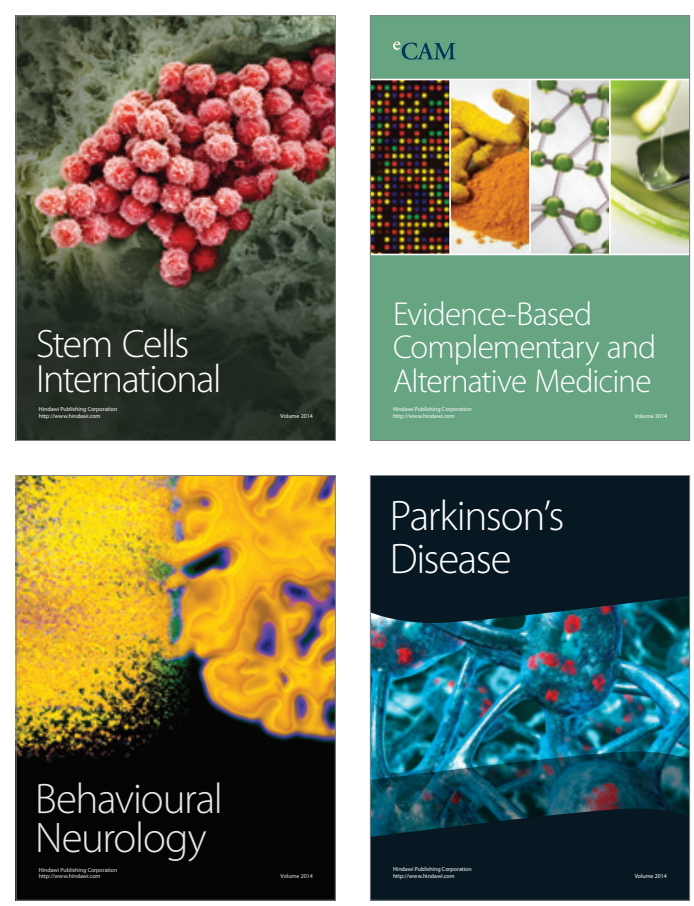

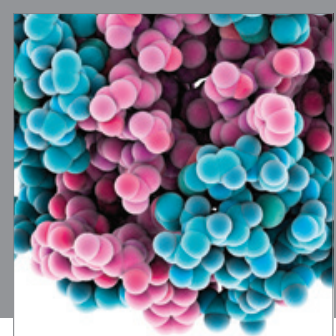

Journal of
Diabetes Research

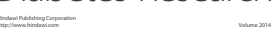

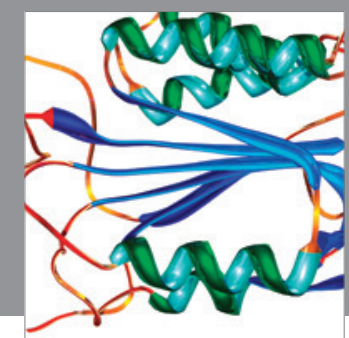

Disease Markers
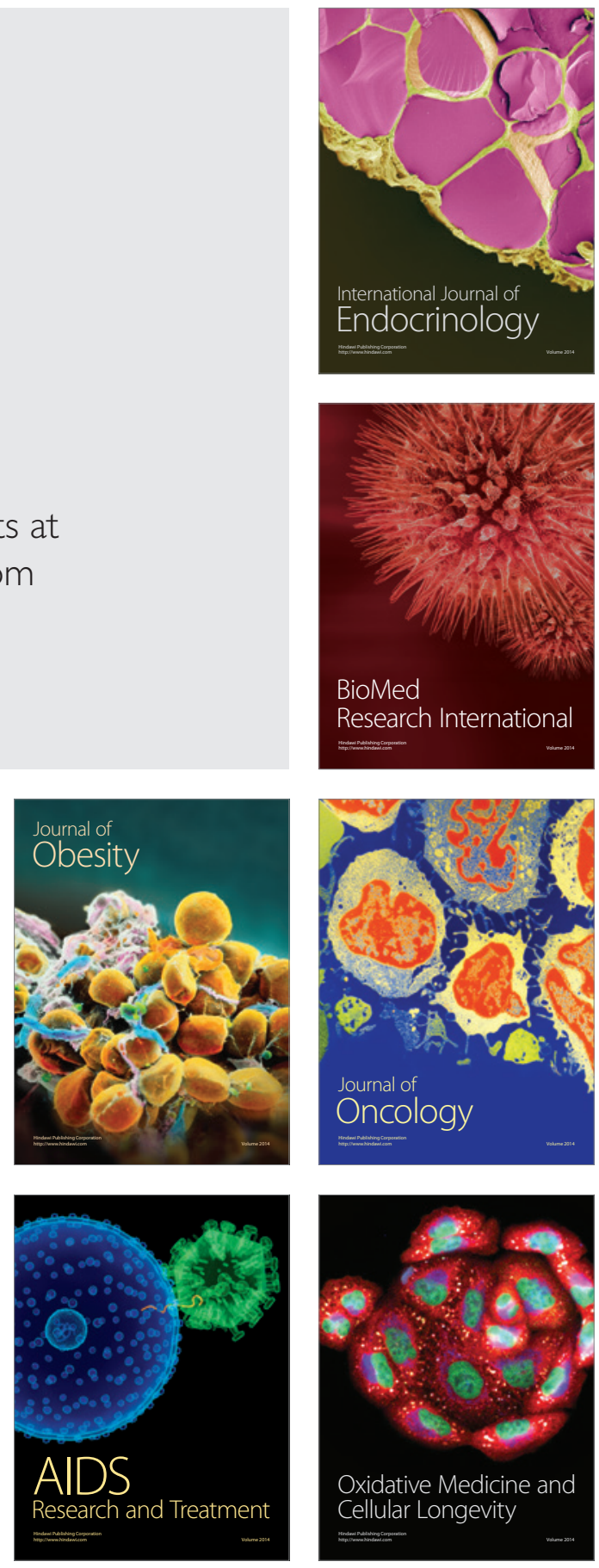